\title{
Autoreactive Antibody Repertoire Is Perturbed in Atherosclerotic Patients
}

\author{
Giuseppina Caligiuri, Dorothea Stahl, Srini Kaveri, Théano Irinopoulous, \\ Françoise Savoie, Chantal Mandet, Marta Vandaele, Michel D. Kazatchkine, \\ Jean-Baptiste Michel, and Antonino Nicoletti
}

INSERM U460 (GC, FS, J-BM) and INSERM U430 (SK, TI, CM, MV, MDK, AN), Paris, France; and University of Münster (DS), Münster, Germany

\begin{abstract}
SUMMARY: In patients with clinical symptoms of coronary atherosclerosis, $T$ cells are activated and directed to autologous proteins contained in the active plaques, suggesting that autoimmune responses may play a role in atherosclerosis progression. Organ-specific autoimmune diseases are sometimes accompanied by broad alterations of serum autoreactive antibody repertoires. We thus investigated antibody repertoires at a global level, using a technique of immunoblotting that allows for the quantitative screening of antibody reactivities in complex antibody mixtures toward a large panel of antigens derived from homologous tissue extracts, followed by multiparametric statistical analysis of the data. We analyzed the autoreactive lgG repertoire in 20 patients with documented coronary atherosclerosis and in 20 matched healthy controls. Total proteins from atherosclerotic carotid specimens and normal arterial tissues (target organs) and from kidney, liver, and stomach (non-target control organs) were used as panels of antigens. Patients had a significantly perturbed antibody repertoire and an enhanced autoreactivity of IgG to target and non-target organs, as compared with controls. Reactivity of purified IgG to plaque and normal artery proteins was greater in patients, but reactivity of IgG in the whole serum toward normal arterial tissue was lower than in controls; this suggests that, in patients, autoreactivity toward normal arteries is regulated by serum factors. Our data indicate that atherosclerotic patients develop a perturbed humoral immune response directed toward arterial proteins, which impacts on the overall autoreactive repertoire. These findings further substantiate that autoimmune processes take place in atherosclerosis and most likely influence disease progression. (Lab Invest 2003, 83:939-947).
\end{abstract}

C oronary atherosclerosis is associated with local (van der Wal et al, 1994) and systemic (Caligiuri et al, 1998) activation of the immune system. Atherosclerotic plaques contain inflammatory cells and activated $T$ cells, suggesting that immune mechanisms are important factors in the pathogenesis of atherosclerosis (Hansson et al, 1989). We (Caligiuri et al, 2000) and others (Liuzzo et al, 2000) have recently shown that the T-cell repertoire of patients with acute coronary syndromes is perturbed. Indeed, we have shown that the T-cell response was antigen-driven and directed to target epitopes contained in the culprit coronary atherosclerotic plaques (Caligiuri et al, 2000).

In addition to cell-mediated immune responses, humoral immune responses are also associated with atherosclerosis (Hansson, 2001). Several self-antigens have been proposed as candidate targets of both the cell-mediated and humoral immune responses (Hans-

\section{DOI: 10.1097/01.LAB.0000077010.90550.FF}

Received October 2, 2002.

G.C. received a grant from EU (BMH4-CT98-5108). D.S. was the recipient of grants from INSERM and CNRS, from the Central Laboratory of the Swiss Red Cross, and the German Society for Transfusion Medicine and Immunohaematology.

Address reprint requests to: Dr. G. Caligiuri, INSERM EMI 00-16, Faculté de Necker, 156, rue de Vaugirard, 75015 Paris, France. E-mail: caligiuri@necker.fr son, 2001). The significance of the reactivity to one or the other self-antigen is complex because selfreactive $T$ cells (Filion et al, 1995) as well as selfreactive $B$ cells and autoantibodies are present in healthy animals and in humans, under physiologic conditions (Coutinho et al, 1995; Hayakawa et al, 1999). Normal serum contains "natural" antibodies of the $\lg G, \lg \mathrm{M}$, and $\lg \mathrm{A}$ isotypes, reactive with a variety of self-antigens including nuclear structures, intracellular and cell surface components, and extracellular molecules (Kazatchkine and Kaveri, 2001) and may participate in a variety of physiologic activities. Nevertheless, most natural antibodies are of the IgG class and they are believed to be essential for the immunoregulatory effects of therapeutic immunoglobulins in immune-mediated disorders (Kazatchkine and Kaveri, 2001). They are called natural antibodies because they are generated in the absence of deliberate immunization and independently of exposure to foreign antigens (Coutinho et al, 1995). Natural antibodies participate in the first line of defense against infectious agents, in the immune regulation, or in the transport of biologically active molecules (Coutinho et al, 1995). The repertoires of natural self-reactive IgG antibodies in the serum of healthy individuals is stable throughout life and is restricted to a limited subset of immunodominant autoantigens that remains conserved with ageing (Lacroix-Desmazes et al, 1995; Mouthon et al, 1995a). Despite extensive research in this field, the 


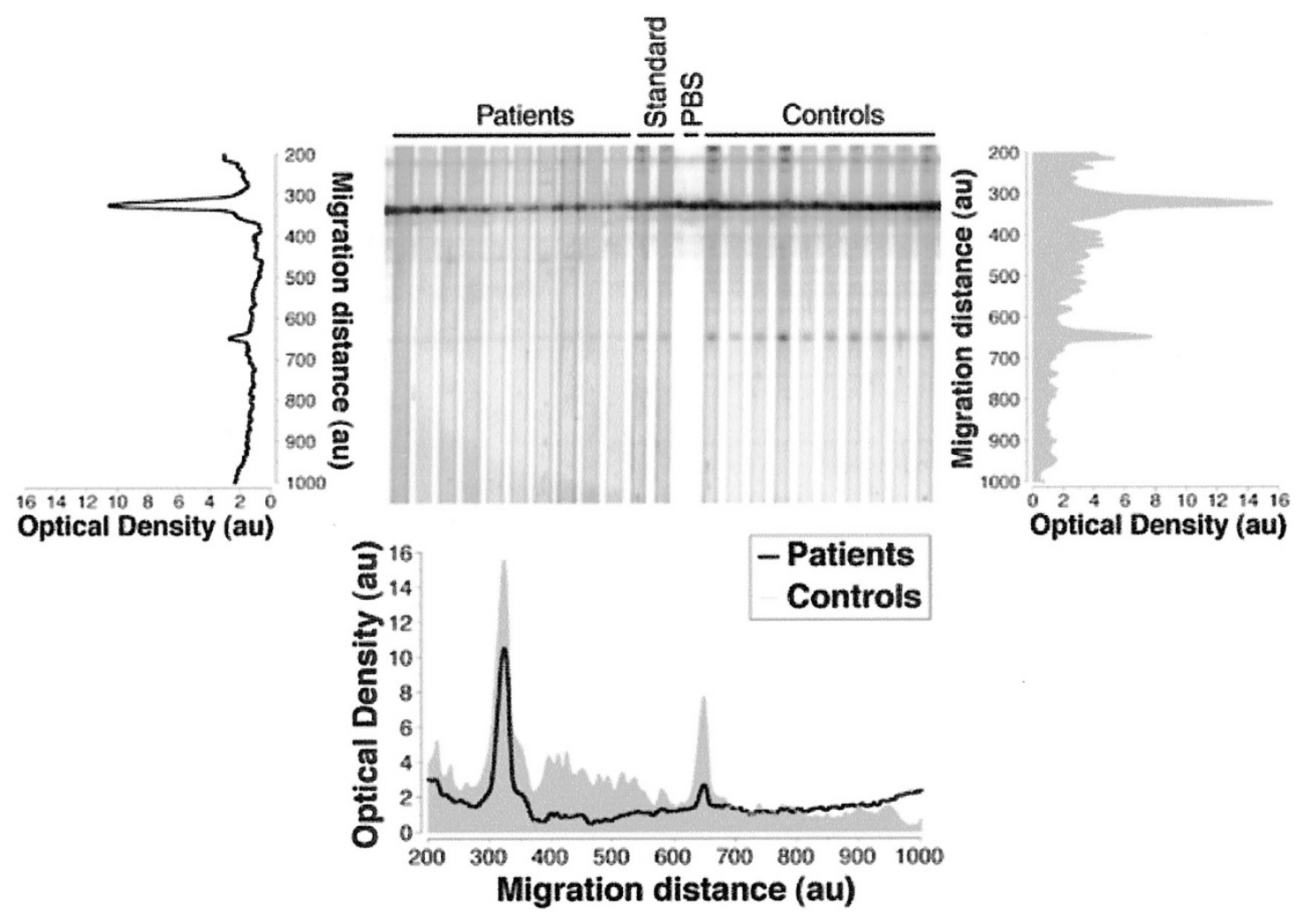

Figure 1.

Quantitative analysis of immunoblots. Representative image of the miniblots used for quantitative immunoblotting analysis of autoreactive antibodies in patients and controls. Total protein extracts from whole organs (mammary artery in the example) were separated by electrophoresis and transferred onto a nitrocellulose membrane. Membranes were incubated with the serum of different individuals (patients, left; controls, right) with the standard $(200 \mathrm{~g} / \mathrm{ml}$ of pooled human lgG) and PBS (negative control). Fixed immunoglobulins (IgG in the example) were then revealed using the appropriate secondary antibody coupled to alkaline-phosphatase. Densitometric profiles of antibody reactivities in the two groups were acquired with a high-resolution CCD scanner. Densitometric profiles were determined by computer-assisted analysis, which allowed the rescaling and quantitative comparison of patterns of reactivity in the two groups (patients vs controls, bottom panel).

relationship between natural autoantibodies and those arising in patients with autoimmune diseases remains unclear (Kazatchkine and Coutinho, 1994).

Immunochemical approaches to analyze repertoires using single antigen targets have the disadvantage of addressing a complex system by reducing it to a collection of single antigen-antibody interactions. This limitation may be overcome by using a recently developed global approach for analyzing antibody repertoires. The approach permits the quantitative screening of antibody reactivities in complex antibody mixture such as serum toward a large panel of antigens derived from tissue extracts by quantitative immunoblotting and interpretation of the data by means of multiparametric statistical analysis (Haury et al, 1994; Mouthon et al, 1995a, 1995b; Nobrega et al, 1993). A possible limitation of this approach concerns the panel of antigens used: reactivities to antigens of very low or very high molecular weight are excluded and only protein antigens are tested, whereas other macromolecules are not analyzed. Such an immunoblotting technique does not primarily aim at distinguishing self-reactive antibody repertoires on the basis of specificity of epitopes but is used to compare the nature of antibody repertoires between groups of individuals by studying global reactivity patterns toward a broad range of self-antigens (Stahl et al, 2000b). This method assumes that reactivities primarily responsible for the autoimmune manifestations will have detectable repercussions on the whole repertoire and implies that detection of alterations of immunoreactivity does not necessarily concern etiologic antigens. Interestingly, application of this quantitative immunoblotting method has demonstrated that repertoires in several autoimmune disorders are altered (Sharshar et al, 1998; Stahl et al, 2000a).

Because most natural autoantibodies in adult serum are of the IgG class (Kazatchkine and Kaveri, 2001), this implies that autoreactive $T$ cells contribute to the repertoire of autoreactive B cells. Given that there are severe perturbations in the T-cell repertoire in atherosclerosis, it is conceivable that these alterations would exert a repercussion on the autoantibody repertoire. To test this hypothesis, we evaluated by quantitative immunoblotting the humoral autoreactivity pattern toward autoantigens in atherosclerotic patients as compared with healthy individuals.

\section{Results}

The Autoreactive Antibody Repertoire to Non-Target Organs Is Perturbed in Atherosclerotic Patients

Patients showed enhanced IgG autoreactivities to all non-target organ extracts, as compared with controls 


\section{pIgG}
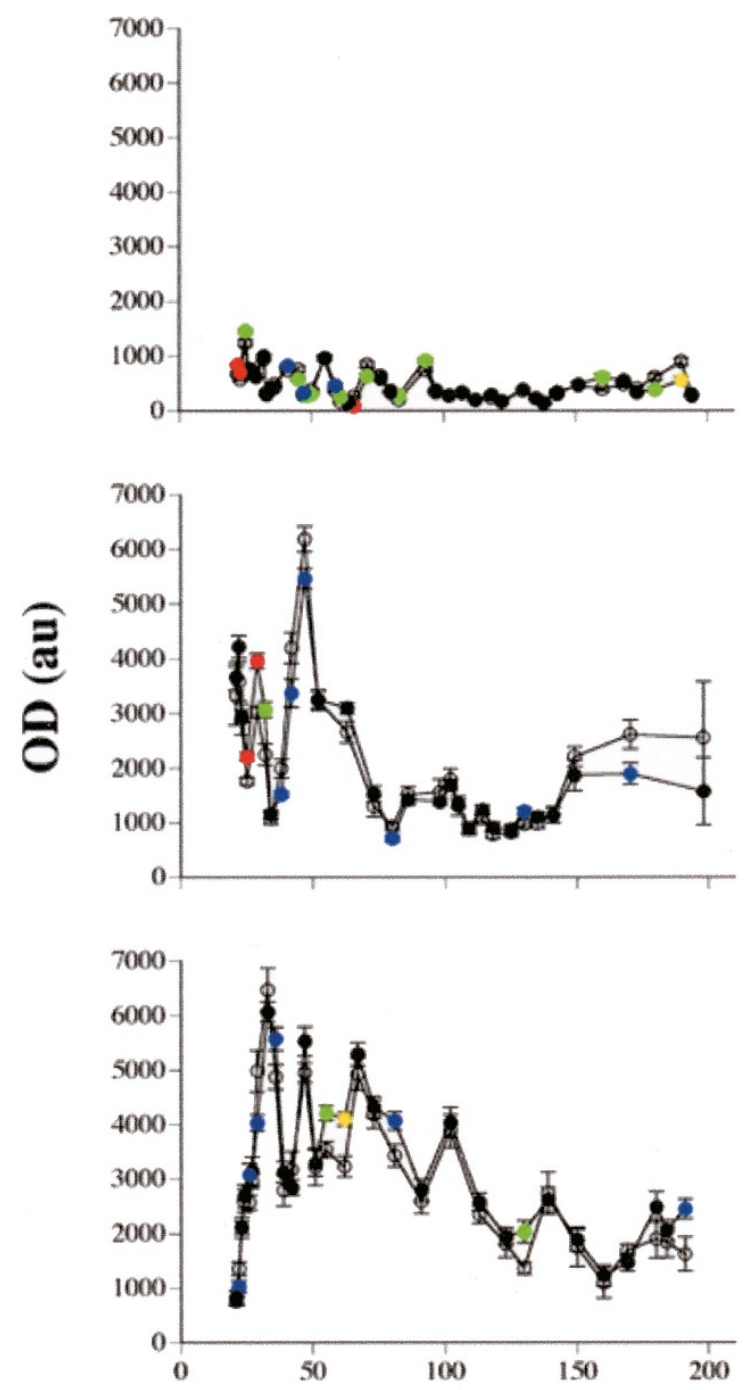

\section{Serum IgG}
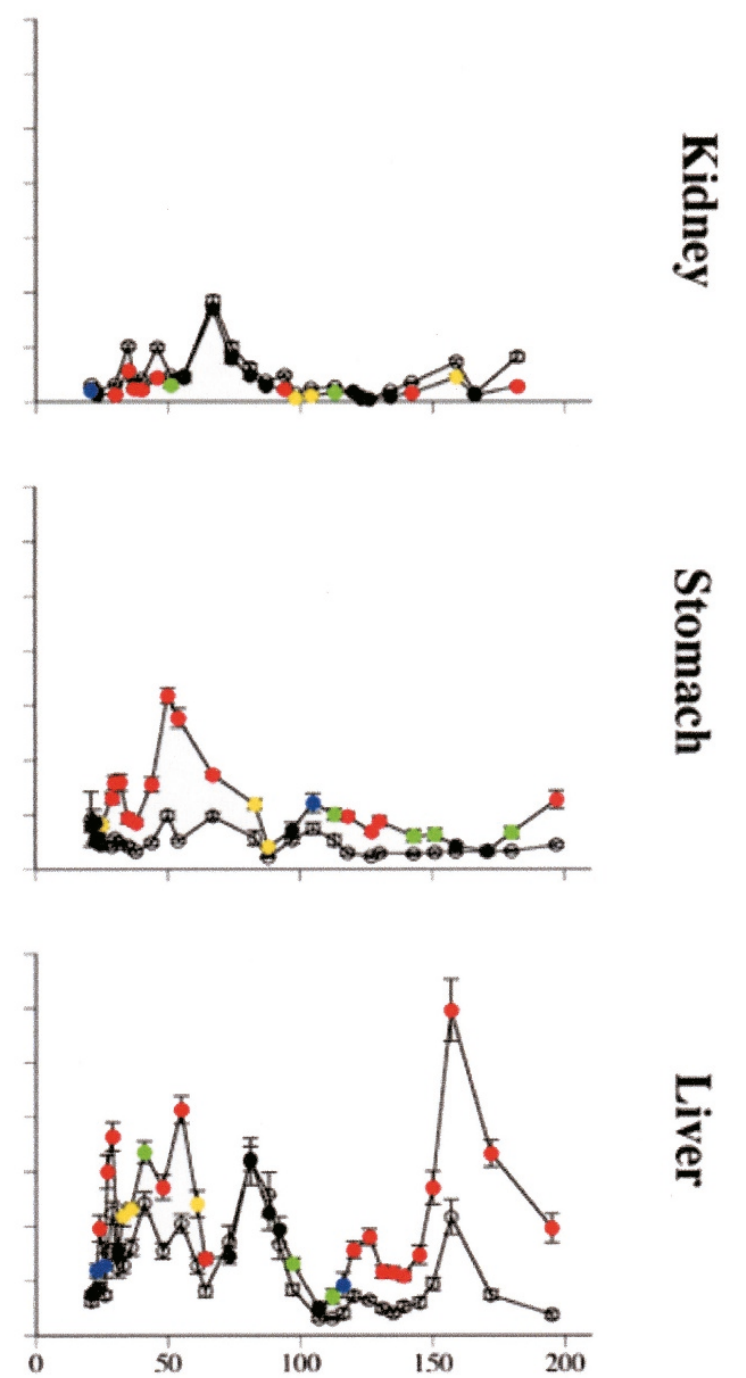

Molecular weight (Kda)

\begin{tabular}{|lll|}
\hline O Controls & $\mathrm{p}<0.0001$ & $\mathrm{p}<0.01$ \\
- Patients & $\mathrm{p}<0.001$ & $\mathrm{p}<0.05$ \\
\hline
\end{tabular}

Figure 2.

Autoreactivity to non-target organs in patients and controls. Densitometric profiles of antibody reactivities in the two groups were submitted to computer-assisted analysis. Data represent mean and SD of the peak reactivity within the sections in the two groups (closed circles = patients; open circles = controls), after subtraction of the reactivity of the secondary antibody alone. Autoreactivities to non-target organs (kidney, stomach, and liver) were significantly enhanced in patients as compared with controls. $O D=$ optical density; au, arbitrary unit.

(Fig. 2). Groups were compared by PCA within a 21- to 57-dimension vector space, depending on the antibody source and tissue extract, and fitted into the 2-dimensional linear subspace that accounted for $40 \%$ to $84 \%$ of the variance and allowed the most powerful discrimination of the groups. PCA data showed that the repertoire of autoreactive antibodies was perturbed in atherosclerotic patients as detected by the lack of homology (group overlapping) in the autoreactive antibody repertoire of patients as compared with controls (Fig. 3). The difference between patients and controls was observed for serum IgG and plgG autoreactive repertoires for control artery as well as for all non-target organs, suggesting that the perturbation in the autoantibody repertoire of atherosclerotic patients is generalized. Intriguingly, patients also showed a higher homogeneity (group clustering) than controls in the whole serum IgG repertoire to normal 

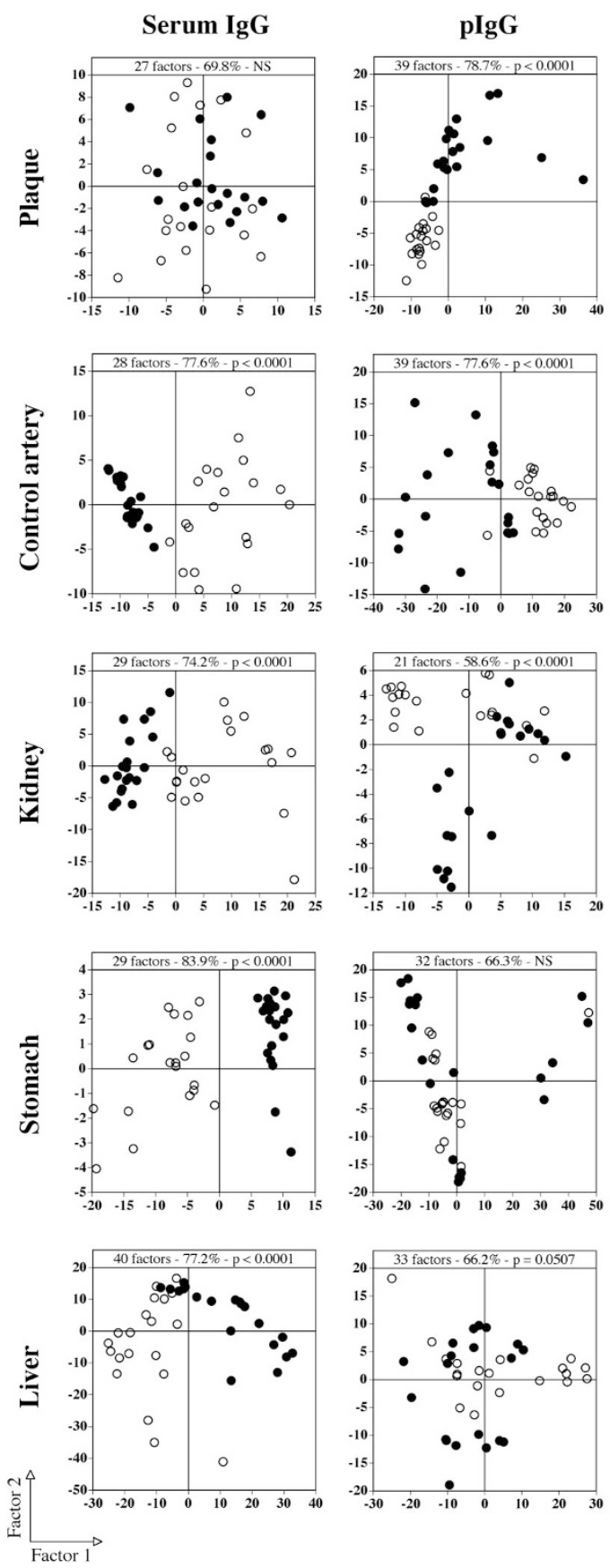

\section{Figure 3.}

Principal component analysis (PCA) of autoantibody repertoire. Multiparametric analysis by PCA allowed the comparison of repertoires of antibody reactivities of individuals in patients as compared with controls. The PCA summarizes multidimensional information (the number of dimensions is equal to the number of peaks of reactivity defined by the sections and cannot therefore be represented graphically if the number of reactivities is greater than 3 ) and finds all the "factors," ie, those associations of variables (in this case the autoantibody reactivities to different protein bands) that can discriminate between groups of individuals. Factors are ranked according to their power to explain the variance between groups. PCA results are here represented in 2-dimensional graph displaying factor 1 vs factor 2, representing together 40 to $83,6 \%$ of the total variance. At the top of each graph is displayed the number of total factors found by PCA, the percentage variance explained by factor $1+$ factor 2 , and the $p$ value. Each symbol represents the repertoire of antibody reactivities of a single individual (closed circles = patients; open circles $=$ controls). organs (Fig. 3). For plaque proteins, the repertoire of serum autoreactive IgG displayed an extremely heterogeneous pattern both in patients and in controls and there was a high degree of homology between the two groups (Fig. 3). The repertoire of plgG autoreactive to target organs (plaque and control artery proteins) was significantly different in patients as compared with controls (Fig. 3), whereas the reactivity to non-target organs was similar in the two groups, with the exception of the kidney (Fig. 3).

\section{Patients' Autoreactive IgG Recognize Specific Protein Bands in Plaque Extracts}

Serum IgG autoreactivity to plaque protein extracts was significantly higher in patients as compared with controls. plgG autoreactivity was particularly increased for protein $\geq 50 \mathrm{kDa}$ (Fig. 4). PCA analysis showed that the autoreactive antibody repertoire to plaque extracts was significantly different in patients and controls (Fig. 3).

\section{Patients' Autoreactive IgG to Normal Arterial Proteins Are Controlled in the Whole Serum}

PCA analysis of autoreactivities to normal arteries discriminated patients and controls (Fig. 3) and plgG autoreactivity to normal arterial tissue was greater in patients than in controls (Fig. 4). However, whole serum IgG autoreactivity was significantly higher in controls as compared with patients (Fig. 4).

\section{Patients' Autoreactive IgG Have a Higher Affinity for Target Organs as Compared with Controls}

Immunohistochemistry imaging showed that both patients' and controls' IgG recognize a wide range of epitopes on the atherosclerotic tissue sections without any evident topologic difference (data not shown). Therefore, we tested the hypothesis that the increased autoreactivity found in patients' immunoblots was a result of a higher affinity of autoreactive plgG. Thus, we coupled plgG from patients with a red fluorochrome and plgG from controls with a blue fluorochrome. These antibody preparations were then used on serial cryosections in double staining at reciprocal dilutions. Analysis of computer-assisted densitometry data showed that plgG from patients could bind to atherosclerotic tissue at lower concentration as compared with controls and equal concentrations could actually displace controls' plgG from the common epitopes (Fig. 5).

\section{Discussion}

Atherosclerosis harbors several features characteristic of autoimmune diseases (Nicoletti et al, 1999; Ross, 1999). We have previously shown the presence of a specific T-cell response in patients with active coronary atherosclerosis (Caligiuri et al, 1998) and that putative antigenic targets for activated $\mathrm{T}$ cells are localized in the autologous plaque (Caligiuri et al, 2000). Furthermore, we (Caligiuri et al, 2000) and 


\section{pIgG}

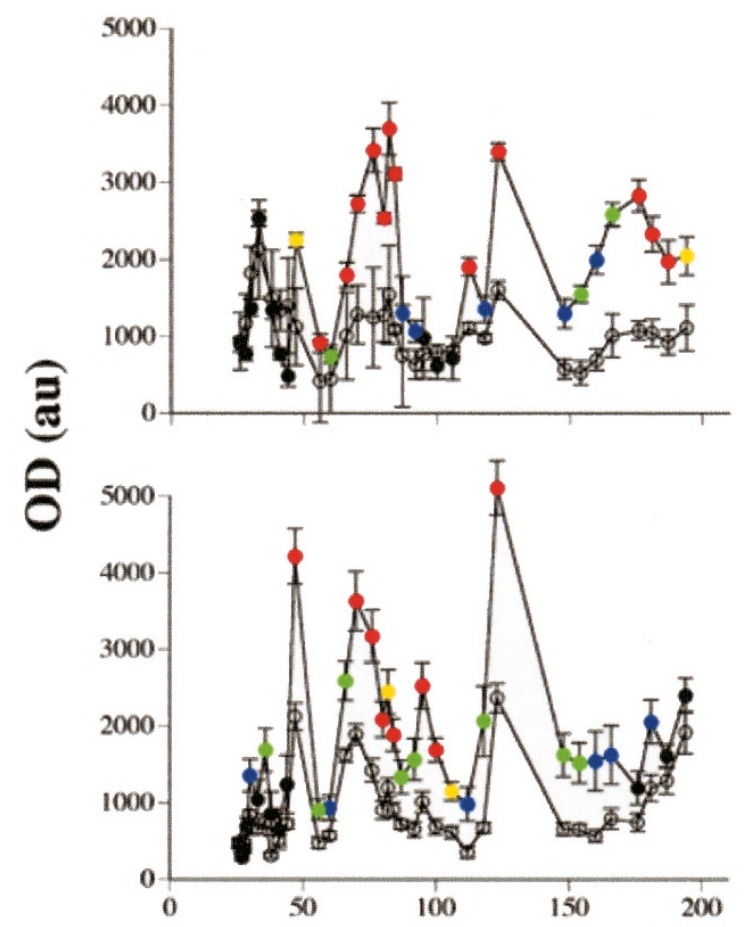

\section{Serum IgG}
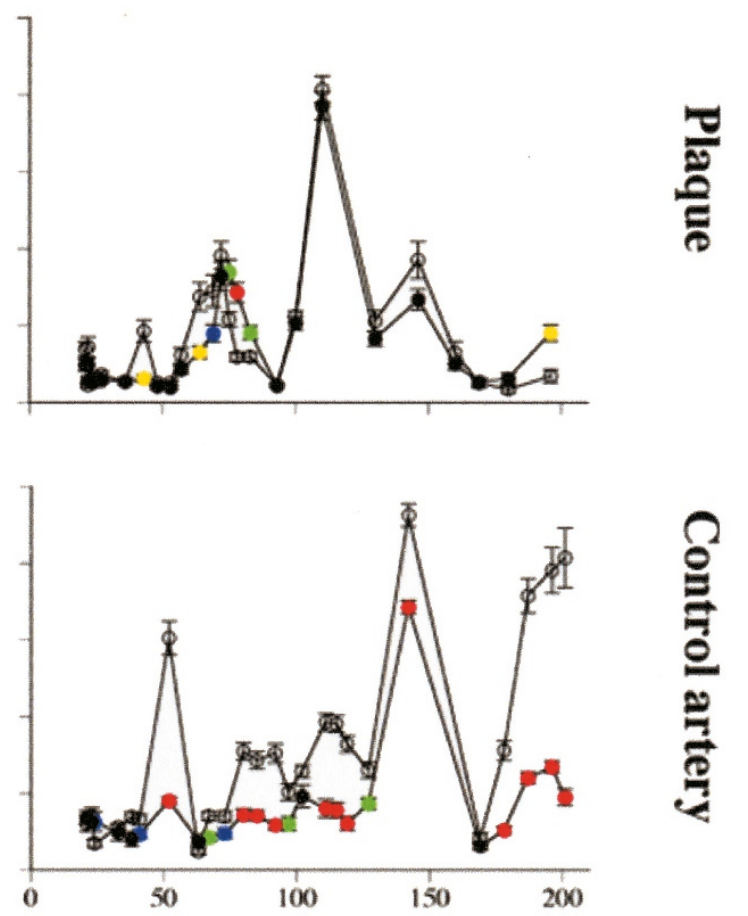

Molecular weight (Kda)

\begin{tabular}{|lll|}
\hline C Controls & $\mathrm{p}<0.0001$ & $\mathrm{p}<0.01$ \\
- Patients & $\mathrm{p}<0.001$ & $\mathrm{p}<0.05$ \\
\hline
\end{tabular}

Figure 4.

Autoreactivity to target organs. Densitometric profiles of antibody reactivities in the two groups were submitted to computer-assisted analysis. Data represent mean and SD of the peak reactivity within the sections in the two groups (closed circles = patients; open circles = controls), after subtraction of the reactivity of the secondary antibody alone. Autoreactivities to target organs (plaque, control artery) were significantly enhanced in patients as compared with controls. $O D=0$ tical density; $a u=$ arbitrary unit.

others (Liuzzo et al, 2000) have shown that the T-cell receptor repertoire is perturbed in patients with acute manifestations of atherosclerotic coronary disease. In the present study, we show that perturbations of the immune response also extend to antibody repertoires. The quantitative immunoblotting approach that we have used shows that the antibody repertoires of atherosclerotic patients displayed severely disturbed antigen recognition profiles similar to those detected in conventional autoimmune disorders. Furthermore, plgG from patients as compared with healthy controls exhibited a higher affinity for common epitopes in target organs. This indicates that the perturbed repertoire of patients is biased toward atherosclerosisspecific epitopes.

First, we found that the variances of individual autoantibody repertoires among each group were similar, indicating that healthy donors and patients with atherosclerosis each represented homogenous groups of individuals. At variance, the autoantibody repertoires of the two groups were discriminated by the PCA, indicating that the antigen panels and/or the intensity with which they were recognized by the antibodies differed between atherosclerotic patients and healthy controls. This was true for most of the antigenic and $\lg$ preparations with the notable and unexpected exception of the serum IgG repertoires to the plaque antigens.

Indeed, we found that whole serum IgG autoreactive repertoires to plaque epitopes were similar in patients and in controls, whereas plgG repertoires were clearly distinct. The reactivity of self-reactive IgG in normal serum is known to be partially masked by several molecular interactions (Adib et al, 1990; Hurez et al, 1993; Mouthon et al, 1995a; Saenko et al, 1992) including (i) the binding of antibodies to soluble antigens; (ii) the concentration- and avidity-dependent competition between autologous IgM and IgG for the binding of the natural antibodies to the self-antigen; and (iii) complementary (anti-idiotypic) interactions be- 

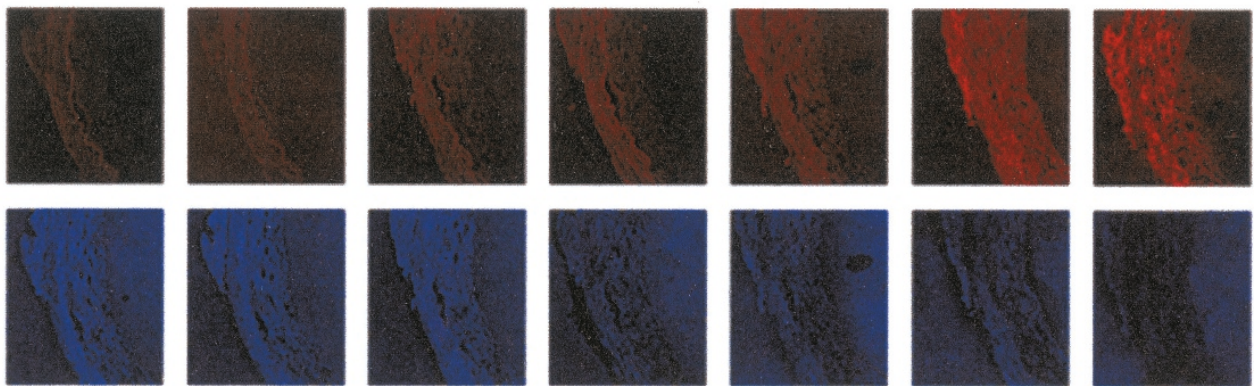

0

0.25

0.50

0.75

1.00

2.00

Binding of:

Patients' IgGs

Controls' IgGs

$\frac{\text { Patient [IgG] }}{\text { Control [IgG] }}$

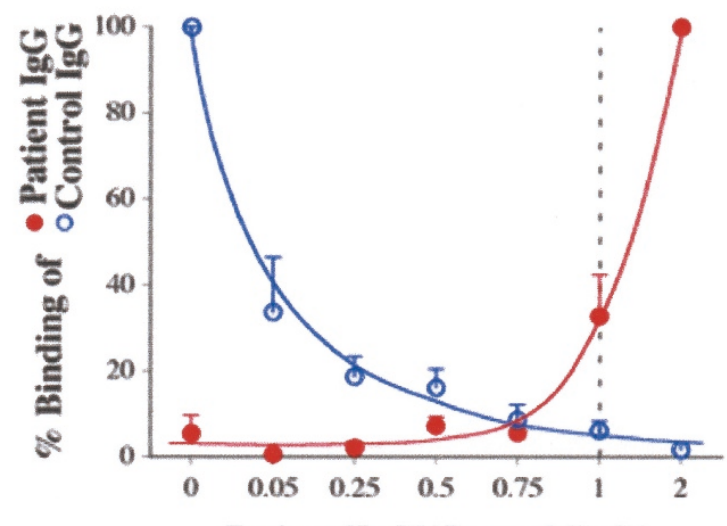

Patient [IgG]/Control [ IgG]

Figure 5.

Competitive binding of patients' and controls' purified lgG (plgG). Patients' plgG showed a significantly greater affinity than controls for target organ epitopes. Representative image of double immunostaining of consecutive control artery sections with different concentrations of antibodies from one patient and a matched control (top). Antibody binding was quantified by fluorescence intensity and densitometric computer-assisted analysis. Percentage of binding to sections for patients (red, $n=20$ ) and controls (blue, $n=20$ ) are displayed according to the ratio of the concentration of IgG from the patient to the concentration of IgG from the control. Dotted line $=$ equal concentration for patients' and controls' IgG $(p<0.001$ patients vs controls $)$.

tween V-regions of autologous immunoglobulins. Thus, the reactivity of plgG toward self-antigens reflects the repertoire of naturally activated self-reactive B cells, whereas the reactivity of unisolated serum IgG represents the net result of autoreactivity in the presence of neutralizing elements of serum. From these observations, it is tempting to speculate that atherosclerotic patients developed antibodies that recognize plaque epitopes as demonstrated by the increased reactivity of their plgG but that the reactivities of these antibodies are controlled by one or several of the mechanisms mentioned above because whole serum IgG from patients behave similar to those of healthy controls. A separate ongoing study is aimed at addressing whether the IgG-dependent autoreactivity is kept under control by self-reactive IgM in atherosclerotic patients.

The results obtained with normal arterial tissue corroborates this idea that autoreactive IgG are kept in check in atherosclerotic patients. Indeed, autoreactivity of whole serum IgG toward healthy arterial tissue was lower in patients than in controls despite the fact that plgG from patients and controls recognized the same protein bands in normal arterial tissue. Again, this suggests that IgG autoantibodies are produced in atherosclerotic patients but that their reactivities are masked in serum.

Most of the protein bands on blots as well as the pattern of epitopes recognized by plgG on organ sections were similar in patients and controls. However, reactivity of patients' plgG was consistently higher. Indeed, we found that patients' autoreactive IgG competed with natural antibodies of healthy controls for binding on the same epitopes on arterial tissue sections, which suggests that patients' autoreactive plgG are characterized by a higher affinity. These antibodies that recognize the same protein bands as the natural antibodies from healthy donors, but with an increased affinity, might originate from maturation of natural autoantibodies.

An insight into the pathogenic role of these antibodies has been provided by two previous studies performed in atherosclerosis-prone apolipoprotein $E$ knockout $\left(E^{\circ}\right)$ mice. In the first study, we treated $E^{\circ}$ mice with a polyclonal Ig (IVIg) preparation (Nicoletti et al, 1998). IVIg preparations consist of intact IgG mol- 
ecules with a distribution of IgG subclasses corresponding to that in normal human serum. They can thus be assumed to contain the entire array of variable (antigen-binding) regions of antibodies in normal serum and provide us with a source of natural antibodies. Injections of $E^{\circ}$ mice with these preparations of natural antibodies were shown to be atheroprotective, possibly through normalization of the humoral repertoires. Concordantly, we have shown in a second study that splenic B cells harbor potent atheroprotective activity (Caligiuri et al, 2002).

Splenectomy aggravated atherosclerosis in $\mathrm{E}^{\circ}$ mice and transfer of splenic $B$ cells from atherosclerotic $E^{\circ}$ mice significantly reduced disease development in young $\mathrm{E}^{\circ}$ mice. Thus, $\mathrm{B}$ cell-associated protective immunity develops during atherosclerosis and bears the intrinsic capacity to reduce disease progression. These observations present an internal paradox. If atherosclerosis is associated with an antiatherogenic humoral immune response, why does it not prevent the onset of the disease? One possible answer is that such immunologic education occurs only after disease exposure, at which time it may be too late to be protective (Witztum, 2002). In contrast, transfer of immunocompetent cells or of natural antibodies to young $\mathrm{E}^{\circ}$ mice provides protection at a much earlier stage in disease progression and at a time when such an intervention is still effective. An alternative explanation provided by the present study is that the protective humoral response is held under control by serum factors preventing its atheroprotective effects in patients.

In conclusion, the present study demonstrates that atherosclerosis is associated with severe generalized perturbations of IgG autoreactivities. This corroborates the previous findings on disturbed T-cell repertoires (Caligiuri et al, 1998, 2000; Liuzzo et al, 2000). Disturbances of autoantibody reactivities toward arterial tissue antigens rely on an increased affinity of IgG for atherosclerosis-specific antigens controlled by intrinsic regulatory mechanisms involving serum factors. Although the panel of self-antigens recognized by patients is similar to that recognized by healthy individuals, the intensity of reactivity of patients' plgG toward atherosclerosis-specific epitopes is significantly higher in patients.

\section{Methods}

\section{Population}

Patients. Twenty patients (age $59 \pm 6$ years; 16 men) with documented coronary atherosclerosis were included in the study.

Controls. Twenty age- and sex-matched apparently healthy subjects were included into the study; physical examination and medical histories did not give any hint of atherosclerotic disease.

Noninclusion Criteria. Fever, recent vaccination, known or suspected autoimmune disease, immunomodulating drug therapy, malignancy, recent $(<3$ months) major trauma or surgery, and anemia. This study has been approved by the local institutional ethical committee. The subjects gave informed consent.

\section{Antibody Preparation}

Autoreactivities were tested using either whole serum or purified IgG (plgG). Serum was obtained from a $10-\mathrm{ml}$ blood sample and stored into aliquots at $-20^{\circ} \mathrm{C}$. Serum levels of $\lg \mathrm{G} 1,2,3,4, \lg \mathrm{A}, \lg \mathrm{M}$, and $\lg \mathrm{E}$ were measured by nephelometry and were similar between the control and patient groups (data not shown). Serum total IgG concentration were adjusted at $200 \mu \mathrm{g} / \mathrm{ml}$ for use in immunoblots. plgG were obtained from separate serum aliquots by affinity chromatography on protein G-Sepharose (HiTrap, Amersham Pharmacia Biotech) following the manufacturer's instructions. Purity of IgG and lack of contaminating $\lg \mathrm{A}$ and $\lg \mathrm{M}$ were assessed by SDS-PAGE and ELISA. The concentration of plgG was determined by spectrophotometry at $280 \mathrm{~nm}$. Intravenous Ig (IVIg; Sandoglobulin, Sandoz), a therapeutic preparation of injectable polyclonal human IgG, was used as positive IgG control.

\section{Quantitative Immunoblot}

We have used a quantitative immunoblotting technique that allows the simultaneous assessment of reactivities of different sources of antibodies with a large number of protein antigens in total tissue extracts (Haury et al, 1994). Sources of autoantigens were extracts of normal human stomach, kidney, and liver (atherosclerosis non-target organs) and mammary artery and carotid endarterectomy obtained during surgical procedures (atherosclerosis target organs). Proteins were extracted on ice in $2 \%$ SDS, 1.45 M 2- $\beta$-mercaptoethanol, $125 \mathrm{~mm}$ Tris/ $\mathrm{HCl}, \mathrm{pH} 6.8$, containing $1 \mu \mathrm{g} / \mathrm{ml}$ aprotinin, $1 \mu \mathrm{g} / \mathrm{ml}$ pepstatin, and 1 mм EDTA. Samples were sonicated four times for 30 seconds to disrupt DNA. The solubilized extracts were then boiled for 5 minutes. After dialysis against PBS, protein concentration was determined (Total Protein Test Kit, Bio-Rad). Proteins were separated on minigels by SDS-PAGE in 10\% polyacrylamide at $20 \mathrm{~mA}$ per gel and transferred onto nitrocellulose membranes (Schleicher \& Schuell) for 60 minutes at $0.8 \mathrm{~mA} / \mathrm{cm}^{2}$ by using a Semi-Dry Electroblotter (Gelman Sciences, Ancos). Nitrocellulose membranes were blocked with PBS containing 0.2\% Tween 20 (Sigma) (Batteiger et al, 1982). Serum and plgG dilutions from 10 patients and 10 controls were incubated with each membrane after addition of one sample per slot in a Cassette Miniblot System (Immunetics) for 4 hours at room temperature. A sample of the reference IgG preparations (standard) was included in each blot, to rescale the different membranes transferred with a given protein extract and to adjust for the intensity of staining of different membranes.

Immunoreactivities were revealed by using alkalinephosphatase rabbit $\left.\mathrm{F}(\mathrm{ab})_{2}\right)_{2}$ anti-human IgG (Dako, Copenhagen) and nitroblue tetrazolium, a bromo- 
chloro-indolyl-phosphate substrate (Promega). Profiles of antibody reactivities in the two groups were acquired by high-resolution scanning densitometry using a macro developed in National Institutes of Health image software (Fig. 1). Computer-assisted analysis of the densitometric profiles allowed the rescaling and quantitative comparison of patterns of reactivity of antibodies from individuals in the two groups. Proteins in areas of the membranes in between the immunoblotted lanes were stained with colloidal gold (Protogold, Biocell) and subjected to a second densitometric analysis to record the protein profile and quantitate transferred proteins. This allowed the immunoreactivity profiles to be compared by referring to their corresponding protein profile corrected for electrophoretic migration defects after superimposing corresponding protein peaks by computer analysis (Stahl et al, 2000b).

\section{Immunohistochemistry}

Topographic analysis of binding of autoreactive IgG to the target organs was performed by immunohistochemistry. plgG from controls was coupled with a blue fluorescent dye (Fluorolink TM-Ab Cy5 labeling kit; Amersham), whereas plgG from patients was coupled with a red fluorescent dye (Fluorolink TM-Ab Cy3 labeling kit; Amersham) following the manufacturer's instructions. Atherosclerotic samples were fixed in PBS/formaldehyde $4 \%$ and embedded in paraffin. To compare the affinity of patients' and controls' plgG for atherosclerotic tissue epitopes, seven consecutive sections were stained with various dilution ratios of patients' and controls' IgG mixtures (ratio patient/ control: 1/0, 1/20, 1/4, 1/2, 1/1.3, 1/1, 2/1). Quantitative analysis of fluorescence was performed by confocal microscopy and a program developed with Qwin (Leica).

\section{Statistical Analysis}

Densitometric data from the blots were analyzed with the software IGOR (WaveMetrics, Lake Oswego, Oregon). The $t$ test (Statview 5.0) was used to compare patient and control peak reactivities. To discriminate between groups of individuals, areas corresponding to peak optical density for each protein preparation were submitted to principal component analysis (PCA) (Jobson, 1992). Results of statistics on PCA data allowed the homology (ie, the clustering or overlapping of the symbols corresponding to individuals in different groups) and the homogeneity (ie, the relative variation of repertoires of antibody reactivities) to be determined.

\section{Acknowledgement}

We are indebted to Prof G. K. Hansson for useful discussions. We thank Dr. V. Frémeaux-Bacchi for valuable help.

\section{References}

Adib M, Ragimbeau J, Avrameas S, and Ternynck T (1990). IgG autoantibody activity in normal mouse serum is controlled by IgM. J Immunol 145:3807-3813.

Batteiger B, Newhall WJ 5th, and Jones RB (1982). The use of Tween 20 as a blocking agent in the immunological detection of proteins transferred to nitrocellulose membranes. J Immunol Methods 55:297-307.

Caligiuri G, Liuzzo G, Biasucci LM, and Maseri A (1998). Immune system activation follows inflammation in unstable angina: Pathogenetic implications. J Am Coll Cardiol 32: 1295-1304.

Caligiuri G, Nicoletti A, Poirier B, and Hansson GK (2002). Protective immunity against atherosclerosis carried by $B$ cells of hypercholesterolemic mice. J Clin Invest 109:745753.

Caligiuri G, Paulsson G, Nicoletti A, Maseri A, and Hansson GK (2000). Evidence for antigen-driven T-cell response in unstable angina. Circulation 102:1114-1119.

Coutinho A, Kazatchkine MD, and Avrameas S (1995). Natural autoantibodies. Curr Opin Immunol 7:812-818.

Filion MC, Bradley AJ, Devine DV, Decary F, and Chartrand P (1995). Autoreactive T cells in healthy individuals show tolerance in vitro with characteristics similar to but distinct from clonal anergy. Eur J Immunol 25:3123-3127.

Hansson GK (2001). Immune mechanisms in atherosclerosis. Arterioscler Thromb Vasc Biol 21:1876-1890.

Hansson GK, Holm J, and Jonasson L (1989). Detection of activated $\mathrm{T}$ lymphocytes in the human atherosclerotic plaque. Am J Pathol 135:169-175.

Haury M, Grandien A, Sundblad A, Coutinho A, and Nobrega A (1994). Global analysis of antibody repertoires. 1. An immunoblot method for the quantitative screening of a large number of reactivities. Scand J Immunol 39:79-87.

Hayakawa K, Asano M, Shinton SA, Gui M, Allman D, Stewart CL, Silver J, and Hardy RR (1999). Positive selection of natural autoreactive B cells. Science 285:113-116.

Hurez V, Kaveri SV, and Kazatchkine MD (1993). Expression and control of the natural autoreactive IgG repertoire in normal human serum. Eur J Immunol 23:783-789.

Jobson JD (1992). Applied multivariate data analysis, vol 2. New York: Springer.

Kazatchkine MD and Coutinho A (1994). Boundaries between physiological autoreactivity and pathological autoimmunity. In: Coutinho A and Kazatchkine MD, editors. Autoimmunity: Physiology and Disease. New York: Wiley-Liss, 243.

Kazatchkine MD and Kaveri SV (2001). Immunomodulation of autoimmune and inflammatory diseases with intravenous immune globulin. N Engl J Med 345:747-755.

Lacroix-Desmazes S, Mouthon L, Coutinho A, and Kazatchkine MD (1995). Analysis of the natural human IgG antibody repertoire: Life-long stability of reactivities towards self antigens contrasts with age-dependent diversification of reactivities against bacterial antigens. Eur J Immunol 25:25982604.

Liuzzo G, Goronzy JJ, Yang H, Kopecky SL, Holmes DR, Frye $\mathrm{RL}$, and Weyand CM (2000). Monoclonal T-cell proliferation and plaque instability in acute coronary syndromes. Circulation 101:2883-2888. 
Mouthon L, Haury M, Lacroix-Desmazes S, Barreau C Coutinho A, and Kazatchkine MD (1995a). Analysis of the normal human IgG antibody repertoire: Evidence that IgG autoantibodies of healthy adults recognize a limited and conserved set of protein antigens in homologous tissues. J Immunol 154:5769-5778.

Mouthon L, Nobrega A, Nicolas N, Kaveri SV, Barreau C, Coutinho A, and Kazatchkine MD (1995b). Invariance and restriction toward a limited set of self-antigens characterize neonatal IgM antibody repertoires and prevail in autoreactive repertoires of healthy adults. Proc Natl Acad Sci USA 92: 3839-3843.

Nicoletti A, Caligiuri G, Paulsson G, and Hansson GK (1999). Functionality of specific immunity in atherosclerosis. Am Heart J 138:438-443.

Nicoletti A, Kaveri S, Caligiuri G, Bariety J, and Hansson GK (1998). Immunoglobulin treatment reduces atherosclerosis in apo E knockout mice. J Clin Invest 102:910-918.

Nobrega A, Haury M, Grandien A, Malanchere E, Sundblad A, and Coutinho A (1993). Global analysis of antibody repertoires. II. Evidence for specificity, self-selection and the immunological "homunculus" of antibodies in normal serum. Eur J Immunol 23:2851-2859.

Ross R (1999). Atherosclerosis: An inflammatory disease. N Engl J Med 340:115-126.
Saenko VA, Kabakov AE, and Poverenny AM (1992). Hidden high-avidity anti-DNA antibodies occur in normal human gammaglobulin preparations. Immunol Lett 34:1-5.

Sharshar T, Lacroix-Desmazes S, Mouthon L, Kaveri S, Gajdos P, and Kazatchkine MD (1998). Selective impairment of serum antibody repertoires toward muscle and thymus antigens in patients with seronegative and seropositive myasthenia gravis. Eur J Immunol 28:2344-2354.

Stahl D, Lacroix-Desmazes S, Heudes D, Mouthon L, Kaver SV, and Kazatchkine MD (2000a). Altered control of selfreactive IgG by autologous IgM in patients with warm autoimmune hemolytic anemia. Blood 95:328-335.

Stahl D, Lacroix-Desmazes S, Mouthon L, Kaveri SV, and Kazatchkine MD (2000b). Analysis of human self-reactive antibody repertoires by quantitative immunoblotting. $\mathrm{J}$ Immunol Methods 240:1-14.

van der Wal AC, Becker AE, van der Loos CM, and Das PK (1994). Site of intimal rupture or erosion of thrombosed coronary atherosclerotic plaques is characterized by an inflammatory process irrespective of the dominant plaque morphology. Circulation 89:36-44.

Witztum JL (2002). Splenic immunity and atherosclerosis: A glimpse into a novel paradigm? J Clin Invest 109:721-724. 